\title{
Social network differences of chronotypes identified from mobile phone data
}

\author{
Talayeh Aledavood ${ }^{1 *}$ (D), Sune Lehmann ${ }^{2,3}$ and Jari Saramäki ${ }^{1}$
}

${ }^{\text {*Correspondence: }}$

talayeh.aledavood@aalto.fi

'Department of Computer Science,

Aalto University, Espoo, Finland

Full list of author information is

available at the end of the article

\section{空 Springer}

\begin{abstract}
Human activity follows an approximately 24-hour day-night cycle, but there is significant individual variation in awake and sleep times. Individuals with circadian rhythms at the extremes can be categorized into two chronotypes: "larks", those who wake up and go to sleep early, and "owls", those who stay up and wake up late. It is well established that a person's chronotype can affect their activities and health. However, less is known about the effects of chronotypes on social behavior, even though many social interactions require coordinated timings. To study how chronotypes relate to social behavior, we use data collected with a smartphone app on a population of more than seven hundred volunteer students to simultaneously determine their chronotypes and social network structure. We find that owls maintain larger personal networks, albeit with less time spent per contact. On average, owls are more central in the social network of students than larks, frequently occupying the dense core of the network. These results point out that there is a strong connection between the chronotypes of people and the structure of social networks that they form.
\end{abstract}

Keywords: Chronotype; Social networks; Mobile phone data; Centrality

\section{Introduction}

Life on Earth follows a circadian rhythm [1-4]. This circadian pattern includes human activities and sleep, with rhythms reflected at the psychological, physiological, and biochemical levels [5, 6]. Even though all humans are diurnal and endogenously controlled by an internal circadian clock, there are individual differences in how the internal clock is synced with the environment's daily rhythm $[7,8]$. These differences can be classified with three chronotypes [9]. At the two extremes are the morning-active people ("larks") and the evening-active people ("owls"), and the rest fall in the intermediate category whose rhythms do not deviate much from the population average (note that there are no absolute criteria for any given chronotype). It has been shown that a person's chronotype can change over the course of her life, but it is fairly stable within time periods of the order of a few years [10].

Chronotypes are not independent traits. There has been a lot of interest in the epidemiology of chronotypes [11], e.g., in terms of age, gender, personality, income, or health risks $[9,12,13]$. Chronotypes have been argued to correlate with certain personal or behavioral traits; these include sociosexual orientation [14], personality (see, e.g., [9]), academic performance [15], body mass index [16, 17], physical and mental health [13, 18-20], and how

(c) The Author(s) 2018. This article is distributed under the terms of the Creative Commons Attribution 4.0 International License (http://creativecommons.org/licenses/by/4.0/), which permits unrestricted use, distribution, and reproduction in any medium, provided you give appropriate credit to the original author(s) and the source, provide a link to the Creative Commons license, and indicate if changes were made. 
people make use of their time [21]. However, the sociology of chronotypes remains less studied, that is, how different chronotypes relate to the structure of social systems.

In this paper, we set out to explore the relationship between chronotypes and social networks. To the best of our knowledge, this is the first study to do so; the connection between chronotypes and social network structure has remained an unexplored area, even though it is known that chronotypes have a social component. This component is due to social cues that act as zeitgebers ("time-givers"), synchronizing the phases of circadian rhythms $[11,22]$. Further, many types of social interactions from face-to-face contacts to phone calls require synchronization and a common understanding of their times. Therefore, it is reasonable to expect that chronotypes and social network structure are correlated, especially if the networks are reconstructed from phone calls as in the present study.

In particular, we study whether the chronotypes of people have effects on (i) the features of their personal (ego-centric) network, and (ii) their positions in their social network in terms of how central they are. We take advantage of a rich dataset of digital activity of students, collected for more than a year at the Technical University of Denmark; we use data on $\sim 700$ individuals. These data allow us to simultaneously estimate the chronotype of each student and to reconstruct the structure of their social network from the metadata of their phone calls and text messages (caller/callee, sender/recipient). In this experiment, participants were equipped with identical smartphones and they volunteered to install an app that collected, among other things, detailed communication metadata as well as times of the phone screen turning on and off. See Materials and Methods for details and Ref. [23] for a full description of the experiment and recorded data.

Mobile phones have become an important part of most people's daily lives, and they are commonly used throughout the day. We take advantage of this to identify chronotypes. Rather than using one of the common questionnaires for identifying chronotypes [24, 25] that may suffer from recall bias, we identify chronotypes from phone usage. Our identification is based on data on the times when the participants' smartphone screens have been switched on (see Materials and Methods for details), using these events as a proxy of a person's activity level. Even though these data do not fully account for a person's activity, the frequency of screen-on events is seen to follow a typical 24-hour cycle where longer periods of inactivity coincide with nights. Because there are clear and persistent individual differences in the levels of early-morning or late-night activity, we use them as a way to assess the chronotypes of individuals [26-28].

To reconstruct the social network of the students, we use data on calls and text messages between the study participants, constructing a network where two participants $i$ and $j$ are linked if there is at least one call or text message from $i$ to $j$ and vice versa. For calculating the total personal network size of each participant (node degree), we also use calls and texts to (anonymized) persons outside the study cohort.

We find that chronotypes correlate with indicators of social behavior. Evening-active owls have larger personal networks than morning-active larks, albeit with less frequent contacts to each network member. From the perspective of the participants' entire social network, owls are more central than larks. This centrality difference is too large to be explained by the differences in personal network size. 


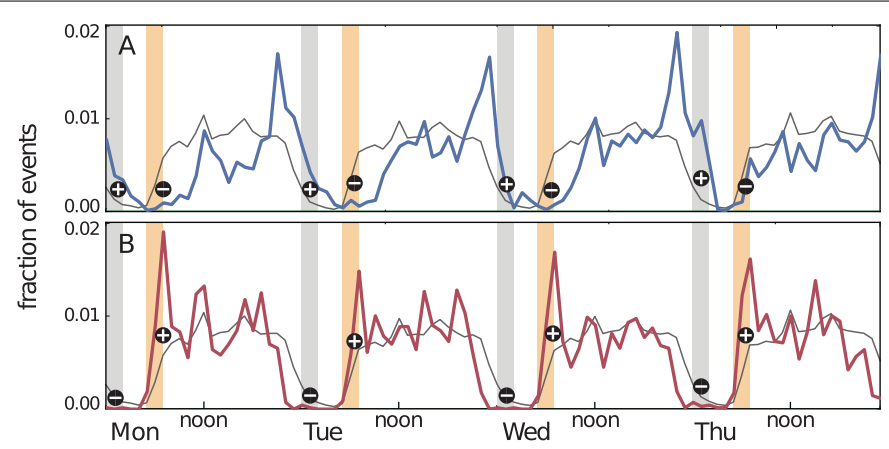

Figure 1 Identifying individuals' chronotypes. The chronotypes of participants are determined by computing the average hourly frequencies of the "screen-on" events of their mobile phones for the first four weekdays. Each participant's pattern is compared to the population average (solid gray line). For chronotype identification, the gray and yellow time ranges are used. If a participant has above-average levels of activity (plus sign) in the night-time gray time range and below-average levels of activity (minus sign) in the yellow morning time range, she is categorized as an evening-active owl (panel (A)). Participants of the opposite pattern (below-average night-time activity and above-average morning activity) are categorized as morning-active larks (panel (B) depicts the pattern for a morning-active person). For details, see Materials and Methods

\section{Results}

\subsection{Screen-on events can be used for chronotype assessment}

We use time-stamped data on "screen-on" events from the smartphone data-collection apps to assign a behavioral chronotype to each participant. Whenever the participant uses the smartphone, from making a call to checking the time, the phone's screen is turned on, and the data-collection app records the time of this event. We use the frequency of these events as a statistical proxy for the daily activity rhythm of the participant, since frequent screen-on events tell that the participant is awake, and night-time event frequencies are typically low or zero. To form an overview of the daily activity patterns of participants, we aggregate the screen-on event frequencies in hourly time bins for the four weekdays from Monday to Thursday for each of the $N=400$ participants who used their phones actively during the whole observation period (see Methods for details; note that for network reconstruction more participants were used). A population-level average rhythm is computed for reference.

Figure 1 shows the screen-on daily rhythms of two study participants (upper and lower panels), together with the population average. The phase of the daily pattern of the student in panel (A) is consistently shifted towards the night, while the student in panel (B) displays a pattern whose phase is shifted towards morning. These phase shifts are captured by the event frequencies in the early morning hours (5 AM to $7 \mathrm{AM}$ ) and late hours of the day (midnight to $2 \mathrm{AM}$ ); "larks" are associated with above-average morning activity and belowaverage night-time activity, and the opposite holds for "owls" (see Methods for details). On this basis, $20 \%$ of the participants $(N=80)$ are labeled as larks, $20 \%$ as owls $(N=80)$, and the rest as intermediate $(N=240)$. These percentages have been chosen to match the literature $[11,29]$ (see Methods). For robustness tests with smaller/larger percentages, see Sect. 2.4. For an illustration of the criteria, see panel (A) in Fig. 2.

In recent years, many studies have tried to unobtrusively measure sleep patterns of individuals by using data collected passively and without any active engagement of the user with the device for the purpose of the study [30-32]. These studies have mostly focused 

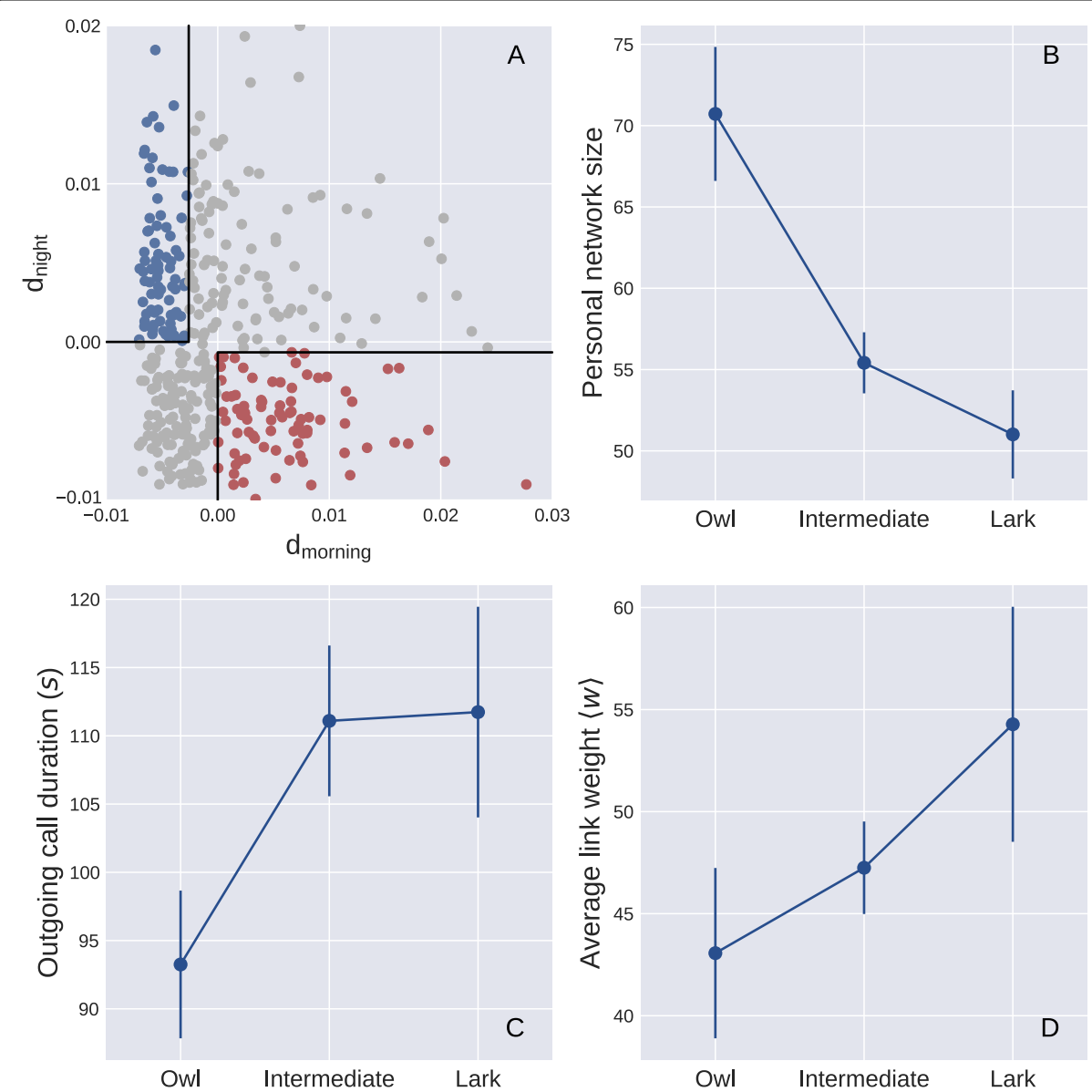

Figure 2 Chronotypes and their behavioral differences. Panel (A) displays the criteria for labeling participants as night-active owls (blue) and morning-active larks (red). $x$ - and $y$-axes represent the difference between morning patterns and evening patterns of each individual's activity compared to the population average activity rhythm, respectively. Panels (B), (C), (D): Average personal network size, average duration of outgoing calls, and average tie strength as measured by communication frequency, for the three different chronotypes

on estimating an individual's different sleep parameters-e.g., sleep duration, mid-sleep time-for each night of sleep during the collection period. Because our focus has been on determining each subject's chronotype, a characteristic that does not change frequently, we have instead used data aggregated over a longer period of time. This makes our results less sensitive to random behavioral variations.

\subsection{Owls have larger personal networks than larks}

We first construct the personal networks of all participants based on both call and text data. For this we use each (hashed and anonymized) phone number that the participant communicates with (through calls and text messages) as a proxy of a social relationship. In this network, each individual is a node and communication events (calls and text messages) between people are the links. The degree of a node (the personal network size) is the total number of people in contact with that node, while the strength of a link is the number of times that it is activated (i.e., the total number of interactions between two nodes). When constructing the personal network of each individual, we consider communication with any phone number, not only those associated with other study participants 
(see Methods). In addition to personal network membership, we count the total number of calls and texts with each contact, as well as the average outgoing call duration. We then study the properties of the personal networks of individuals of each chronotype separately for which we use outgoing calls and text messages.

The average personal network sizes for students of each chronotype are shown in Fig. 2(B). It is evident that owls have personal networks that are much larger than those of larks, with the intermediate chronotype positioned in between (owls: network size $k=70.7 \pm 4.1$, larks: $k=51.0 \pm 2.7$, intermediate $k=55.4 \pm 1.9$ ). When the average call durations and total frequencies of calls and texts per social contact are considered, an opposite trend becomes visible (Fig. 2(C) and (D)): owls make the shortest calls on average and their communication frequency per social tie is the lowest as compared to the intermediate chronotype and in particular to larks. More detailed analysis indicates that owls' calls are on average shorter than those of other chronotypes at all times of day except at night where the differences are within standard errors (mornings owls/larks: 83/100; afternoons owls/larks 86/103; evenings owls/larks 106/141; nights owls/larks 68/64; all numbers in seconds). This reflects the known sub-linear scaling between node degree and strength in social networks (see, e.g., [33]); the larger the number of relationships, the less time is available for each of them. There are also differences in the numbers of screen-on events for the different chronotypes: the mean of number of screen-on events in weeks $2-51$ is 19,829 for larks, 23,509 for members of the intermediate chronotype, and 25,140 for owls.

\subsection{Owls are more central than larks in the social network of participants}

In order to study the network centrality of each participant, we constructed the social network of participating students, so that two individuals $i$ and $j$ are connected with an unweighted link if there are either calls or text messages from $i$ to $j$ and from $j$ to $i$ (see Methods for details). This network consists of $N=734$ participants; out of these, 366 had enough screen-on events to be assigned a chronotype (for filtering criteria, see Methods). We then computed the values of various network centrality measures for all individuals within each of the three chronotypes. The chosen measures were (i) betweenness centrality, measuring the number of shortest paths through a network node, (ii) closeness centrality, quantifying the inverted average geodesic distance to other nodes, (iii) eigenvector centrality, reflecting the level of connectivity to high-centrality nodes in an iterative fashion, and (iv) core number, indicating membership in a core where all nodes are linked to other member nodes with at least $k$ links.

These four centrality measures are displayed in Fig. 3 (panels (A)-(D)), together with a visualization of the network (panel (E)). There is an increasing trend in centrality from larks to owls for all centrality measures: owls are much more central than larks in the network. This is also reflected in the network visualization: owls (blue) are more frequently located in central parts of the network than larks (red).

To test whether the centralities of owls and larks differ only because their degrees differ, we used the so-called configuration model (see Sect. 4.6). It randomizes the structure of the network while retaining the degrees and chronotypes of nodes. We applied the configuration model as the null model and tested whether the ratios of centralities of owls to larks are the same in the null model as observed in the real network. We found that the higher degrees of owls do not alone explain their higher centralities (Fig. 4). For all centrality measures, the real owl-lark ratios lie 5 to 12 standard deviations away from the null 

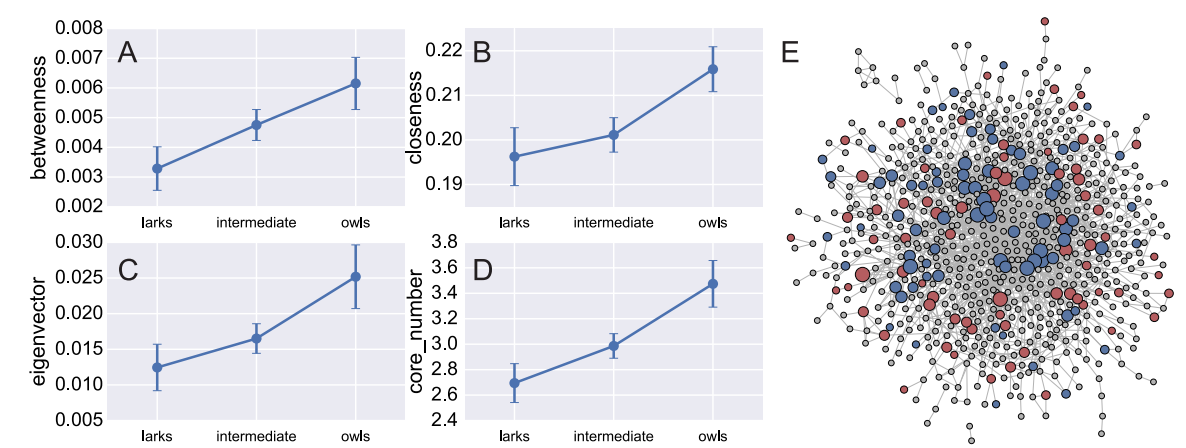

Figure 3 Chronotypes and centrality measures. Chronotypes are associated with network centrality such that owls are on average more central. Panels (A)-(D): network centrality measures (betweenness centrality, closeness centrality, eigenvector centrality, and core number) for the three chronotypes. In all cases, values for the owl chronotype are the highest. Panel (E): a network visualization of the social network of participants. Individuals of the owl (lark) chronotype are displayed as blue (red). The rest are gray. For blue and red nodes, the size is determined by core number. Nodes of the owl chronotype are more frequent in the central core of the network

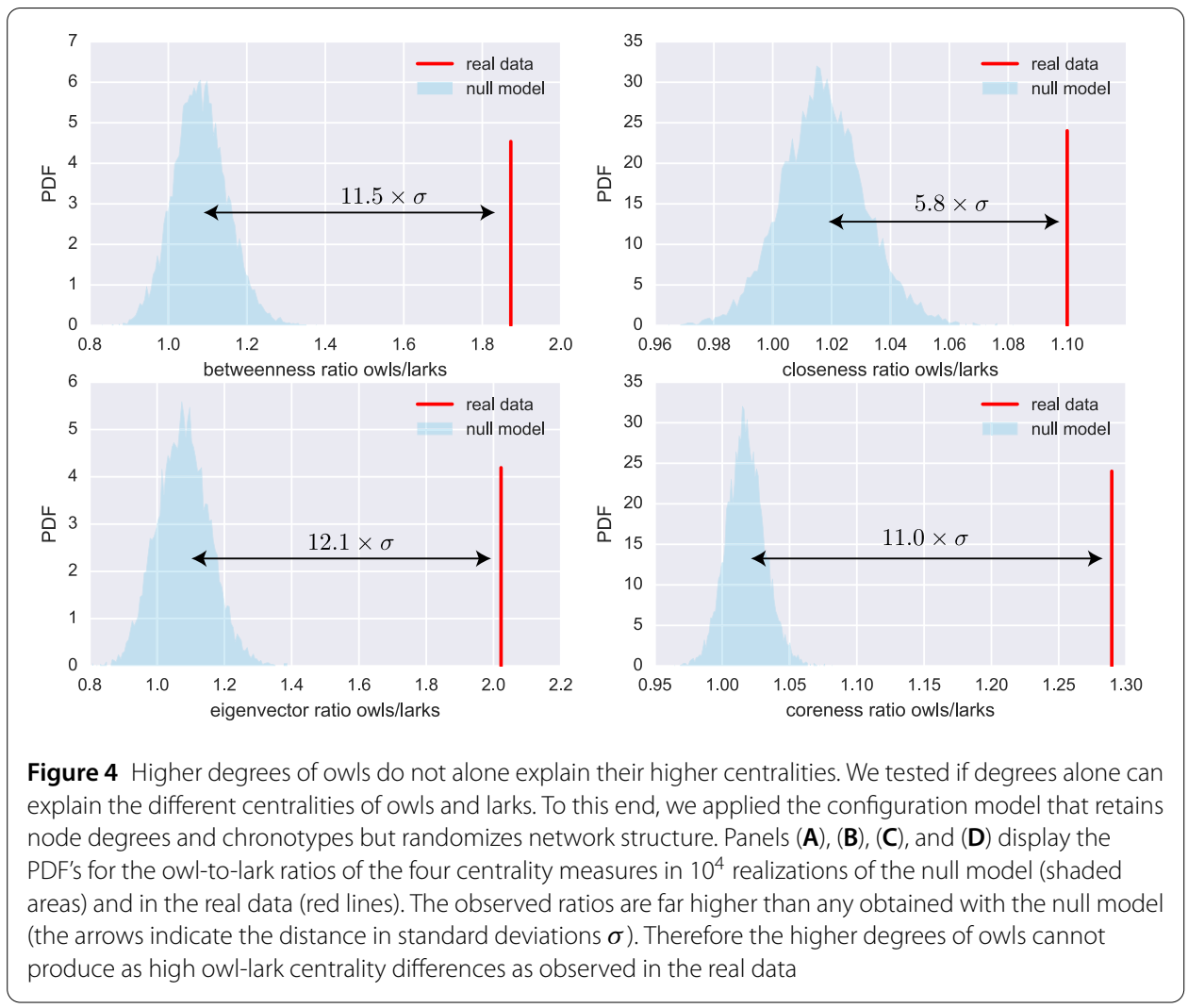

model mean. As an example, in the real data, the average eigenvector centrality of owls is twice higher than that of larks. In the reference model, however, the corresponding factor is only 1.07 on average. This means that there is a small effect from the higher degrees of owls (the ratio is 1.07 instead of 1), but it cannot explain the observed factor of two. To summarize, in the $N=10^{4}$ runs of the configuration model, we never observed owl-to-lark centrality ratios as high as in the original network, for any centrality measure. Therefore, 

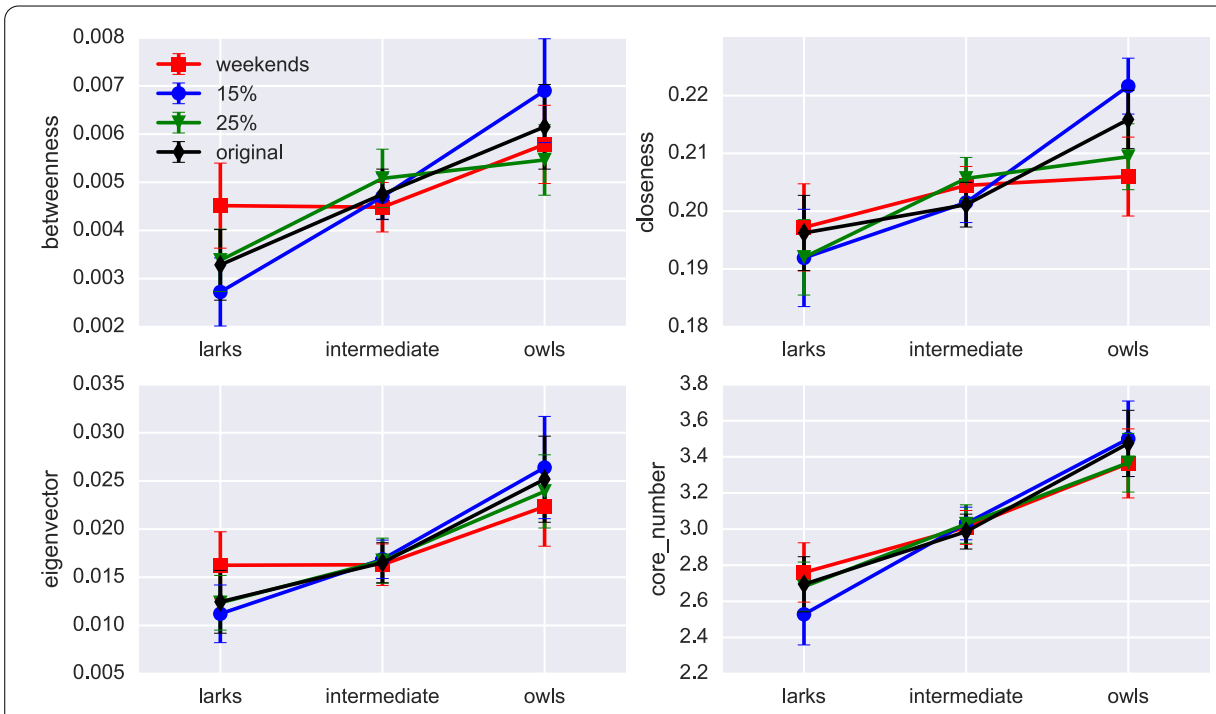

Figure 5 Robustness of centrality measures. We tested whether the centrality results are robust against variations in the analysis pipeline. The four measures are shown above, for the original chronotypes (black diamonds), for chronotypes computed using weekend data only (red squares), and for chronotypes with smaller shares of owls/larks (15\%, blue circles) and larger (25\%, green triangles)

the null hypothesis can be rejected: degrees are not enough to explain why the centralities of chronotypes differ.

\subsection{Robustness of results}

To test how robust our conclusions are against variations in the analysis pipeline, we have recomputed the results with different parameterizations. In particular, we have determined the students' chronotypes (1) using data from a shorter time range (half a year instead of the whole year), (2) from screen-on event frequencies from Friday to Sunday instead of Monday to Thursday, (3) with stricter filtering criteria, taking into account only students who have at least 280 screen-on and screen-off events in each week of the study $(N=222)$, and (4) using smaller and larger percentages of larks and owls (15\% and $25 \%$ instead of the $20 \%$ on which the results so far have been based).

The outcome of the above has been that while there are minor variations in the exact numbers, our results qualitatively hold for all cases (1) to (4). The results for centrality measures are shown in Fig. 5.

We have also tested the behavior of eigenvector centrality using edge weights and separately constructed networks from calls and text messages, with the logarithms of their numbers as weights. Eigenvector centrality is suited for working with weights (unlike the other three). The rationale behind keeping the networks separate is that the numbers of calls and texts measure different things: one conversation may take one call but a large number of text messages. The rationale behind taking the logarithm is that the distributions of these numbers are very broad and without the log, the highest-weight link dominates the entire measure. However, with log weights, in terms of eigenvector centrality, owls are most central and larks least central in both call and text-message networks.

Finally, we have also tested our main results with a method based on Non-negative Matrix Factorization (NMF) for chronotype identification (T.A., S.L., J.S., manuscript in preparation); our results hold when using this method as well. 


\section{Discussion}

There are well-known differences between human chronotypes that have been related to various personal and behavioral traits $[9,34,35]$. redIn this study, we have focused on the social dimension of chronotypes and investigated how the chronotypes of people are reflected in their personal network structure and position in social networks. We have used detailed behavioral data collected from volunteer students with smartphone apps in an experiment at the Technical University of Denmark. We have estimated the chronotypes of students from the times of "screen-on" events, and constructed their social network using mutual calls and text messages as proxies of social relationships. We have shown that evening-active owls have larger personal networks than morning-active larks. Owls are also more central in the social network of students; this difference cannot be explained only by their larger personal network size.

Even though chronotypes are partially regulated by social cues, the link between people's chronotypes and their social networks has been underexplored. Because our results show for the first time that chronotypes are connected to social network structure, it becomes clear that they deserve more attention in studies of social networks and spreading processes on networks; one should not only focus on other well-studied traits such as age, gender or other socio-demographic features. The connection between social networks and chronotypes also calls for more attention to social aspects and cues in studies of chronotypes.

It has been shown in the literature that there is a correlation between the evening-active chronotype and extroversion (see [9] for a review). One might reasonably expect that these correlations translate into properties of personal networks such that owls maintain larger communication networks. Additionally, in [36] the authors suggest that in a social network of adolescents sleep behavior, and in particular sleep loss, spreads in the network. These results further hint towards a potential relationship between sleep behavior and social network properties.

Further, the need to synchronize social activities and common social norms may explain the observed differences in personal network size and network centrality. Social gatherings, including student parties, often take place in the late hours of the day. Because social ties are created and maintained in such events that are more suitable for owls, it is perhaps not surprising that there is a bias in favor of the evening-active chronotype. In contrast, early-morning social gatherings are not common, and it is therefore possible that those with a very early chronotype spend more of their time alone, as there are fewer opportunities for social interaction during their day.

People's chronotypes have traditionally been identified using questionnaires. It has been suggested that questions related to sleeping and waking up habits can explain most of the variance in distinguishing chronotypes [9]. This is also the rationale behind our method: screen-on events are used as proxy of activity, and early-morning and night-time activity levels determine the chronotype. The specific time intervals have been determined as the time ranges where population-level average activity has only started to increase from very low values (mornings), or is about to cease for the night. We have also taken differences between weekend and weekday patterns into account, focusing on weekdays where the students have approximately similar schedules; the literature suggests that owls in particular develop a sleep deficit during the week and therefore their weekend patterns may differ as they compensate for this deficit [37]. 
The data we use were not originally collected for determining or studying chronotypes. As we used the data for this purpose retrospectively, there is no way of validating the obtained chronotypes against common methods such as questionnaires-this would require an experiment of its own. However, it is reasonable to assume that if an individual persistently displays above-average levels of early-morning activity and below-average levels of night-time activity, this pattern would be captured with a similar outcome by questionnaires developed for assessing chronotypes. In general, the possibility of retroactively determining people's chronotypes from time-stamped data opens a lot of possibilities for research, especially together with our observations that chronotypes are not independent of social network structure (or vice versa). There are many datasets available to researchers from time-stamped mobile telephone calls $[38,39]$ to email records that allow for retrospectively determining chronotype and reconstructing social networks [27].

\section{Materials ans methods}

\subsection{Experimental data and its filtering}

In this work, we used data from a large-scale data collection study, in which 1000 identical mobile phones were distributed among students at Technical University of Denmark (DTU) in 2013. This data collection experiment was designed to measure various aspects of social behavior and human dynamics. In the study, smartphone apps were used to collect data with high temporal resolution from various mobile phone sensors. These data were augmented with additional questionnaires that all students in the study filled out. The data collection continued for more than a year; all participants were, however, not active throughout the whole period. A detailed description of this dataset, types of collected data, and research envisioned by means of the dataset are described in [23]. In the present paper, data from weeks 2-51 of year 2014 are used. Weeks 1 and 52 are excluded, because the former starts in the end of 2013 and the latter coincides with end-of-the-year holidays, which may result in untypical activity patterns. The number of participants that used their phone during this year is $N=804$. We apply filters to the data to only include participants who use their phone actively and exclude those who have very little activity or are inactive for a part of the year. The inclusion criteria were: (1) the participant should be active on $80 \%$ of the days, (2) during the weeks included in the study (weeks 2-51), the participant should on average have 280 screen-on and screen-off events. After applying these filters, the total number of participants included for further analysis is $N=400$. The data and the associated code used for this paper will be made available to researchers upon request.

\subsection{Computing the activity patterns of students}

For computing the activity patterns of individuals, one week is divided to one-hour bins. As a result, we have a total of $7 \times 24=168$ bins. For each of the 400 active participants, we go through all screen-on events in the selected study period and assign each event to one of the bins. This way, all events for one person throughout the year are aggregated. These aggregated counts are then normalized so that their values sum to unity. This results in a weekly activity pattern for each person.

\subsection{Identifying chronotypes from activity patterns}

Weekly activity patterns of individuals are used as the basis of assessing chronotypes. Only weekdays from Monday to Thursday were used in the analysis. The reason for this is that 
the literature suggests that individuals may have different behaviors during working days and weekends, and the extent of these differences may vary from one chronotype to another $[37,40]$.

We find the population's average weekly pattern by computing the average of the patterns of all 400 participants. Then, for each person, their weekly pattern is compared with the population average in the early hours of the day (5 AM-7 AM) as well as the late hours (midnight-2 AM). To label an individual as a lark, her pattern should lie above the population average in the early hours, and in addition, in the late hours the difference between the individual's pattern and the population average should be less than 0.00066 . The same applies to identifying owls: at late hours of the night, their pattern should be above the population average and in the early morning hours the difference of the individual's rhythm and the population average should be less than 0.0026 . The numerical thresholds for the differences between activity patterns are selected so that $20 \%$ of the population is labeled as larks and $20 \%$ as owls. These percentages have been chosen to match the literature [11, 29]. The time intervals for early-morning and night-time hours have been selected based on the times when the population average rhythm falls at night and when it begins to rise again in the morning.

\subsection{Reconstructing the social network of students}

Students in the study were all from the same university and mostly began their studies at the same time. To construct the students' social network, all individuals in the study who had communication events during the year 2014 were selected $(N=776)$. In panel (E) of Fig. 3, the social links between students in the study is depicted. Out of the 776 students in the network, 393 had an identified chronotype; 7 students with an identified chronotype were not part of the network because they did not have any calls or text messages. For all panels in Fig. 3, incoming and outgoing calls and text messages were used. However, only those social ties were included that were associated with at least one event in both directions (incoming and outgoing), to assure that a tie between two individuals is representative of a social relationship. This left us with $N=734$ students in the network.

The dataset also contains calls and text messages between study participants and people from outside the study cohort. This makes it possible to build a more comprehensive picture of each individual's personal network based on communication events. For the 393 students with known chronotypes, personal network are built from all outgoing calls and texts. There are a total of 20,841 social links for the 393 students; again we only kept social links which were active at least once in each direction. The properties of the personal network shown in Fig. 2 are based on outgoing communication only.

\subsection{Centrality measures and $k$-shells}

The notion of centrality of a network node can be defined in several ways. In this work, different centrality measures are used: betweenness centrality, eigenvector centrality, and closeness centrality. Betweenness centrality is a proxy of the importance of flows in the network, and it is defined on the basis of the number of shortest paths that a node is a part of; the more shortest paths go through a node, the higher its betweenness centrality. The betweenness centrality $C_{b}(i)$ for node $i$ can be formulated as

$$
C_{b}(i)=\sum_{j<k} \frac{\sigma_{j k}(i)}{\sigma_{j k}},
$$


where $\sigma_{j k}$ accounts for the total number of shortest paths from node $j$ to node $k$ and $\sigma_{j k}(i)$ is the number of those paths which go through node $i$.

Closeness centrality quantifies how far each node is from all other nodes in the network. The closeness centrality $C_{c}(i)$ for node $i$ is defined as

$$
C_{c}(i)=\frac{1}{\left\langle l_{i}\right\rangle}=\frac{N-1}{\sum_{i \neq j} d_{i j}},
$$

where $\left\langle l_{i}\right\rangle$ is the average length of shortest paths of node $i$ to all other nodes in the network, $d_{i j}$ is the shortest path between nodes $i$ and $j$, and $N$ is the total number of nodes in the network.

Eigenvector centrality is an iterative centrality measure that does not only depend on how well-connected each node is, but also takes into account the centrality of its neighbors, such that a node with a small number of central neighbors may outrank one with more less central neighbors [41]. Therefore, it measures how well-connected each node is to other well-connected nodes. Formally, eigenvector centrality of a node is defined as the corresponding element of the eigenvector of the network's adjacency matrix that corresponds to its largest eigenvalue.

The other measure we use is the core number which is defined based on the concept of $k$-cores. $k$-cores are maximal subgraphs in the network where all nodes are connected to other nodes in the subgraph with at least $k$ links. For a node, the core number is the largest value of $k$ for $k$-cores that contains the node [42].

\subsection{Null model for centrality measures}

For reference, we applied the configuration model that randomly rewires the network while keeping the degrees of nodes fixed (and retaining their chronotypes). This model is designed for testing whether some property of nodes can be explained by their degrees alone. We investigated whether the higher centralities of owls are caused only by their higher degrees. To this end, we formulated the null hypothesis as "the ratio of the \{betweenness, closeness, eigenvector, coreness\} centrality of owls to the corresponding centrality of larks is fully explained by the degrees of owls and larks." In other words, the null model posits that chronotypes do not affect the network positions of nodes, only their degrees. If the null hypothesis were true, the owl-lark centrality ratios observed in the original network would not be significantly higher than the ratios observed in the randomized networks. To test the hypothesis, we ran the configuration model $N=10^{4}$ times, and stored the centrality ratios for each run. Their distribution was then compared to the observations.

\section{Acknowledgements}

The authors thank the following people for their help and support at different stages of this work: Ilkka Kivimäki, Bjorn Sand Jensen, Rasmus Troelsgård, Richard Darst, Radu Gatej, and Onerva Korhonen. TA acknowledges the support of EIT Digital doctoral school for her visit to DTU.

Funding

JS and TA were funded by the Academy of Finland, project DigiDay, No. 297195. The funding agencies had no role in design of the research and the data analysis and interpretation. 
Availability of data and materials

An anonymized version of the data used for this work can be made available upon request.

\section{Competing interests}

The authors declare that they have no competing interests.

\section{Authors' contributions}

SL collected the data. TA, JS, and SL designed the research. TA and JS analyzed the data. TA, JS, and SL wrote the paper. All authors read and approved the final manuscript.

\section{Author details}

'Department of Computer Science, Aalto University, Espoo, Finland. '2Department of Applied Mathematics and Computer Science, Technical University of Denmark, Kongens Lyngby, Denmark. ${ }^{3}$ The Niels Bohr Institute, University of Copenhagen, Copenhagen, Denmark.

\section{Publisher's Note}

Springer Nature remains neutral with regard to jurisdictional claims in published maps and institutional affiliations.

Received: 24 April 2018 Accepted: 16 October 2018 Published online: 24 October 2018

\section{References}

1. Panda S, Hogenesch JB, Kay SA (2002) Circadian rhythms from flies to human. Nature 417(6886):329-335

2. Dibner C, Schibler U, Albrecht U (2010) The mammalian circadian timing system: organization and coordination of central and peripheral clocks. Annu Rev Physiol 72:517-549

3. Czeisler CA, Duffy JF, Shanahan TL, Brown EN, Mitchell JF, Rimmer DW, Ronda JM, Silva EJ, Allan JS, Emens JS et al (1999) Stability, precision, and near-24-hour period of the human circadian pacemaker. Science 284(5423):2177-2181

4. Edery I (2000) Circadian rhythms in a nutshell. Physiol Genomics 3(2):59-74

5. Czeisler CA, Weitzman ED, Moore-Ede MC, Zimmerman JC, Knauer RS et al (1980) Human sleep_-its duration and organization depend on its circadian phase. Science 210(4475):1264-1267

6. Edgar RS, Green EW, Zhao Y, van Ooijen G, Olmedo M, Qin X, Xu Y, Pan M, Valekunja UK, Feeney KA et al (2012) Peroxiredoxins are conserved markers of circadian rhythms. Nature 485(7399):459-464

7. Horne JA, Östberg O (1977) Individual differences in human circadian rhythms. Biol Psychol 5(3):179-190

8. Kerkhof GA (1985) Inter-individual differences in the human circadian system: a review. Biol Psychol 20(2):83-112

9. Adan A, Archer SN, Hidalgo MP, Di Milia L, Natale V, Randler C (2012) Circadian typology: a comprehensive review. Chronobiol Int 29(9):1153-1175

10. Lee JH, Kim IS, Kim SJ, Wang W, Duffy JF (2011) Change in individual chronotype over a lifetime: a retrospective study. Sleep Med Res (SMR) 2(2):48-53

11. Roenneberg T, Kuehnle T, Juda M, Kantermann T, Allebrandt K, Gordijn M, Merrow M (2007) Epidemiology of the human circadian clock. Sleep Med Rev 11(6):429-438

12. Tankova I, Adan A, Buela-Casal G (1994) Circadian typology and individual differences. A review. Pers Individ Differ 16(5):671-684

13. Roenneberg T, Merrow M (2016) The circadian clock and human health. Curr Biol 26(10):432-443

14. Jankowski KS, Díaz-Morales JF, Vollmer C, Randler C (2014) Morningness-eveningness and sociosexuality: evening females are less restricted than morning ones. Pers Individ Differ 68:13-17

15. Preckel F, Lipnevich AA, Schneider S, Roberts RD (2011) Chronotype, cognitive abilities, and academic achievement: a meta-analytic investigation. Learn Individ Differ 21(5):483-492

16. Yu JH, Yun C-H, Ahn JH, Suh S, Cho HJ, Lee SK, Yoo HJ, Seo JA, Kim SG, Choi KM et al (2015) Evening chronotype is associated with metabolic disorders and body composition in middle-aged adults. J Clin Endocrinol Metab 100(4):1494-1502

17. Arora T, Taheri S (2015) Associations among late chronotype, body mass index and dietary behaviors in young adolescents. Int J Obes 39(1):39-44

18. Gaspar-Barba E, Calati R, Cruz-Fuentes CS, Ontiveros-Uribe MP, Natale V, De Ronchi D, Serretti A (2009) Depressive symptomatology is influenced by chronotypes. J Affect Disord 119(1):100-106

19. Wong PM, Hasler BP, Kamarck TW, Muldoon MF, Manuck SB (2015) Social jetlag, chronotype, and cardiometabolic risk. J Clin Endocrinol Metab 100(12):4612-4620

20. Merikanto I, Englund A, Kronholm E, Laatikainen T, Peltonen M, Vartiainen E, Partonen T (2014) Evening chronotypes have the increased odds for bronchial asthma and nocturnal asthma. Chronobiol Int 31(1):95-101

21. Kauderer S, Randler C (2013) Differences in time use among chronotypes in adolescents. Biol Rhythm Res 44(4):601-608

22. Allebrandt KV, Teder-Laving M, Kantermann T, Peters A, Campbell H, Rudan I, Wilson JF, Metspalu A, Roenneberg T (2014) Chronotype and sleep duration: the influence of season of assessment. Chronobiol Int 31(5):731-740

23. Stopczynski A, Sekara V, Sapiezynski P, Cuttone A, Madsen MM, Larsen JE, Lehmann S (2014) Measuring large-scale social networks with high resolution. PLoS ONE 9(4):95978

24. Horne JA, Ostberg O (1975) A self-assessment questionnaire to determine morningness-eveningness in human circadian rhythms. Int J Chronobiol 4(2):97-110

25. Roenneberg T, Wirz-Justice A, Merrow M (2003) Life between clocks: daily temporal patterns of human chronotypes. J Biol Rhythms 18(1):80-90

26. Aledavood T, López E, Roberts SG, Reed-Tsochas F, Moro E, Dunbar RI, Saramäki J (2015) Daily rhythms in mobile telephone communication. PLoS ONE 10(9):0138098

27. Aledavood T, Lehmann S, Saramäki J (2015) Digital daily cycles of individuals. Front Phys 3:73. https://doi.org/10.3389/fphy.2015.00073 
28. Aledavood T, López E, Roberts SG, Reed-Tsochas F, Moro E, Dunbar Rl, Saramäki J (2016) Channel-specific daily patterns in mobile phone communication. In: Proceedings of ECCS 2014. Springer, Cham, pp 209-218

29. Levandovski R, Sasso E, Hidalgo MP (2013) Chronotype: a review of the advances, limits and applicability of the main instruments used in the literature to assess human phenotype. Trends psychiatry psychother 35(1):3-11

30. Chen Z, Lin M, Chen F, Lane ND, Cardone G, Wang R, Li T, Chen Y, Choudhury T, Campbell AT (2013) Unobtrusive sleep monitoring using smartphones. In: Proceedings of the 7 th international conference on pervasive computing technologies for healthcare. ICST (Institute for Computer Sciences, Social-Informatics and Telecommunications Engineering), Venice, pp 145-152

31. Jayarajah K, Radhakrishnan M, Hoi S, Misra A (2015) Candy crushing your sleep. In: Adjunct proceedings of the 2015 ACM international joint conference on pervasive and ubiquitous computing and proceedings of the 2015 ACM international symposium on wearable computers. ACM, New York, pp 753-762

32. Cuttone A, Bækgaard P, Sekara V, Jonsson H, Larsen JE, Lehmann S (2017) Sensiblesleep: a Bayesian model for learning sleep patterns from smartphone events. PLoS ONE 12(1):0169901

33. Onnela J-P, Saramäki J, Hyvönen J, Szábo G, Argollo de Menezes M, Kaski K, Barabási A-L, Kertész J (2007) Analysis of a large-scale weighted network of one-to-one human communication. New J Phys 9:179

34. Murnane EL, Abdullah S, Matthews M, Kay M, Kientz JA, Choudhury T, Gay G, Cosley D (2016) Mobile manifestations of alertness: connecting biological rhythms with patterns of smartphone app use. In: Proceedings of the 18th international conference on human-computer interaction with mobile devices and services. ACM, New York, pp 465-477

35. Althoff T, Horvitz E, White RW, Zeitzer J (2017) Harnessing the web for population-scale physiological sensing: a case study of sleep and performance. In: Proceedings of the 26th international conference on World Wide Web, pp 113-122. International World Wide Web Conferences Steering Committee

36. Mednick SC, Christakis NA, Fowler JH (2010) The spread of sleep loss influences drug use in adolescent social networks. PLOS ONE 5(3):9775

37. Wittmann M, Dinich J, Merrow M, Roenneberg T (2006) Social jetlag: misalignment of biological and social time. Chronobiol Int 23(1-2):497-509

38. Blondel VD, Decuyper A, Krings G (2015) A survey of results on mobile phone datasets analysis. EPJ Data Sci 4(1):10

39. Saramäki J, Moro E (2015) From seconds to months: an overview of multi-scale dynamics of mobile telephone calls. Eur Phys J B, Condens Matter Complex Syst 88(6):1-10. https://doi.org/10.1140/epjb/e2015-60106

40. Roenneberg T (2012) Internal time: chronotypes, social jet lag, and why you're so tired. Harvard University Press, Cambridge

41. Newman ME (2008) The mathematics of networks. In: The new palgrave encyclopedia of economics. Palgrave Macmillan, UK, pp 1-12

42. Carmi S, Havlin S, Kirkpatrick S, Shavitt Y, Shir E (2007) A model of Internet topology using k-shell decomposition. Proc Natl Acad Sci 104(27):11150-11154

\section{Submit your manuscript to a SpringerOpen ${ }^{0}$ journal and benefit from:}

- Convenient online submission

- Rigorous peer review

- Open access: articles freely available online

- High visibility within the field

- Retaining the copyright to your article

Submit your next manuscript at $\gg$ springeropen.com 